Trinity University

Digital Commons @ Trinity

Engineering Faculty Research

Engineering Science Department

2011

\title{
Evaporation of Sessile Drops under Combined Diffusion and Natural Convection
}

Peter Kelly-Zion

TrinityUniversity, plkelly@trinity.edu

Christopher J. Purcell

Trinity University, cpursell@trinity.edu

Sawan Vaidya

Trinity University

Jaskirat Batra

Trinity University

Follow this and additional works at: https://digitalcommons.trinity.edu/engine_faculty

Part of the Engineering Commons

\section{Repository Citation}

Kelly-Zion, Peter; Purcell, Christopher J.; Vaidya, Sawan; and Batra, Jaskirat, "Evaporation of Sessile Drops under Combined Diffusion and Natural Convection" (2011). Engineering Faculty Research. 3.

https://digitalcommons.trinity.edu/engine_faculty/3 


\title{
Evaporation of Sessile Drops under Combined Diffusion and Natural Convection
}

\author{
P.L. Kelly-Zion ${ }^{\mathrm{a}, *}$, C.J. Pursell ${ }^{\mathrm{b}}$, S. Vaidya ${ }^{\mathrm{a}}$, J. Batra $^{\mathrm{a}}$ \\ ${ }^{a}$ Engineering Science Department, Trinity University, San Antonio, TX 78212, USA \\ ${ }^{b}$ Chemistry Department, Trinity University, San Antonio, TX 78212, USA
}

\begin{abstract}
Experiments were conducted to investigate the range of applicability of a commonly used assumption for evaporation models of sessile drops, that the transport mechanism that controls the evaporation is vapor diffusion. The evaporation rates of sessile drops of 3-methylpentane, hexane, cyclohexane, and heptane were measured. The radius of the drop contact line was constant during the measurements and drops of radius from $1 \mathrm{~mm}$ to $22 \mathrm{~mm}$ were studied. It was found that a diffusion-controlled evaporation model underpredicts the evaporation rate from $36 \%$ to $80 \%$ depending on the drop size. The increase in the evaporation rate was attributed to a second transport mechanism, natural convection of the vapors, and an empirical model was developed for conditions of combined diffusive and convective transport. Over the broad range of volatilities and drop sizes studied, the evaporation rates computed using the combined transport model agree with the measured values with less than $6 \%$ root mean square error.
\end{abstract}

Keywords: evaporation, diffusion, natural convection, sessile drop, hydrocarbon

\section{Introduction}

The evaporation of sessile drops on solid substrates continues to be the subject of ongoing research not only due to its intrinsic scientific value but

\footnotetext{
${ }^{*}$ Corresponding author

Email address: peter.kelly-zion@trinity.edu (P.L. Kelly-Zion)
} 
also because evaporation plays an important role in many practical applications, e.g. coating, painting and printing. Modeling the dynamics of a sessile drop as the drop first expands to wet the surface and then recedes due to the loss of volume from evaporation has been accomplished recently with very good qualitative agreement between the model and measurements of drop contact line radius and contact angle $[1,2,3,4,5,6,7]$. Good success has also been achieved in modeling the dynamics of a sessile drop which is pinned to a flat horizontal substrate $[8,9,10,11]$. In the case of a pinned drop, the radius of the contact line remains constant until sufficient volume is lost due to evaporation to cause the contact line to pull from its original position as the contact area of the drop reduces. Models for both wetting and pinned drops assume that the evaporation is quasi-steady and the rate is controlled by the rate that vapor diffuses away from the liquid surface. The purpose of this study is to investigate the applicability of the diffusion-controlled evaporation assumption for sessile drops and to develop an empirical evaporation model for conditions in which both diffusion and natural convection are important.

As discussed by many authors, the problem of quasi-steady, diffusionlimited evaporation of a drop is governed by the steady Laplace equation. As reported by Thomas and Ferguson in 1917, Stefan was the first to obtain a solution for the evaporation of a flat, circular surface and to demonstrate that the evaporation rate was proportional to the radius of the surface, and not the area [12]. Stefan's solution was obtained by analogy to the electrostatic case of a charged conductor. Using a similar electrostatic analogy, expressions have been derived for the evaporation rate of a sessile drop for two cases, one in which the contact angle remains constant and the contact area reduces, and the second case in which the contact area remains constant, i.e. a pinned drop, and the contact angle reduces $[8,11,13,14]$. For both of these cases, the rate of evaporation was found to be proportional to the radius of the contact line, in agreement with Stefan's result. According to these solutions, the local evaporative flux is low but increases gradually moving from the center of the drop toward the contact line. The flux becomes large in the region of the contact line and, in fact, one of the practical difficulties of these analytical solutions is that the flux becomes infinite along the contact line.

Despite the fact the flux becomes infinite at the contact line, the flux may be integrated over the surface of the drop to compute the overall evaporation rate. Other approaches that have been taken to model the evaporation of sessile drops are to use a smoothing function or a separate evaporation model 
in the region of the contact line $[2,3,4,5,6,7,8,11,14]$, or to use an empirically derived constant of proportionality to relate the total evaporation rate to the transient radius of the drop contact line $[1,3,15]$. Researchers also have worked to improve the expression for the evaporation rate of sessile drops by accounting for effects such as evaporative cooling [11, 15] and parabolic surfaces [11].

While the direct proportionality between the total evaporation rate and the contact line radius has been experimentally validated for both wetting and pinned sessile drops undergoing diffusion-controlled evaporation, in the words of Starov and Sefiane, 'its demonstration from the theoretical point of view as well as the physical phenomenon behind it remain unclear' [15].

It should be noted that the vast majority of the experimental validations of the quasi-steady, diffusion-controlled evaporation model have been accomplished with drops having a radius less than $3 \mathrm{~mm}$. However, Poulard et al compared the results of experiments with sessile drops having a radius of up to $8 \mathrm{~mm}$ with those of smaller drops and reported a modest difference in the behavior of the transient drop radius as a function of drop size [6]. They concluded that the sensitivity of their solution to the capillary number, which contains an evaporation rate parameter, needs to be 'smoothed out'. A much longer time ago, Thomas and Ferguson measured the evaporation rates of water contained in circular pans of radius from 21 to $100 \mathrm{~mm}$ and determined that on average the evaporation rate varied with the radius raised to the power of 1.69 [12]. For those experiments, the liquid surface was 7 $\mathrm{mm}$ below the rim of the pan, and so the water in their experiments cannot be considered sessile drops. Still, their results call into question the applicability of a diffusion-controlled model for the evaporation of large drops. In the same paper, the authors report that as the depth of the liquid surface from the rim of the pan increases, the dependence of the evaporation rate approaches the square of the radius, which is expected for one-dimensional diffusion.

It is interesting to consider what the range of applicability of the quasisteady, diffusion-controlled evaporation model is. Contrary to the results derived from the solution to the steady Laplace equation, the evaporative flux along the contact line is finite. Therefore, as the radius of the contact line increases, the ratio of the drop surface area to the contact line length increases, and consequently it is reasonable to expect that at some point the evaporation rate would become proportional to the drop radius raised to a power greater than 1 . For very large radii, the evaporation rate may 
be expected to become proportional to the square of the radius (area). Furthermore, it is interesting to consider how the vapor density above the drop may limit the applicability of the diffusion-controlled evaporation assumption. For a vapor density that is either very low or very high in comparison to the ambient gas density, one would expect natural convection to influence the rate of vapor transport from the drop surface and thereby affect the evaporation rate.

In a previous study, schlieren imaging was used to view the vapor clouds that formed over pinned hydrocarbon drops of radius $6.5 \mathrm{~mm}$ [16]. The schlieren videos show the vapor clouds flowing over the surface of the drops indicating the occurrence of natural convection. Further, by modifying the geometry of the substrate surrounding the drop, the relative influences of diffusion and convection were adjusted. That study demonstrated the potential for natural convection to increase the evaporation rate compared to diffusion-limited evaporation. However, drop size was not varied and so the relationship between evaporation rate and drop radius was not determined.

The influences of drop size and vapor density on the evaporation rate are the subjects of this study. The goal is to better define the range of applicability of the diffusion-controlled evaporation model and to provide insight into the evaporation process for cases in which natural convection may be significant.

\section{Materials and Methods}

The evaporation rates of pinned, sessile drops in an initially quiescent atmosphere were measured for a wide range of drop sizes. The ambient temperature and pressure were $23.2{ }^{\circ} \mathrm{C} \pm 0.7^{\circ} \mathrm{C}$ and 1 atm. Four hydrocarbon components were used: 3-methylpentane (3MP), hexane, cyclohexane, and heptane. 3MP and hexane are geometrical isomers and cyclohexane approximately is a third isomer. These isomers have nearly equal molar masses and heats of vaporization but significantly different equilibrium vapor pressures. Heptane was included in the study in order to expand the range of volatilities so that the equilibrium vapor pressures vary by a factor of four. Natural convection is driven by a density difference, and the difference in densities of the vapor-air mixture at the surface of the drop, $\rho_{m}$ (assuming a saturated

mixture) and the surrounding air, $\rho_{a}$, i.e. $\left(\rho_{m}-\rho_{a}\right)$ varies by a factor of three for the components in this study. 


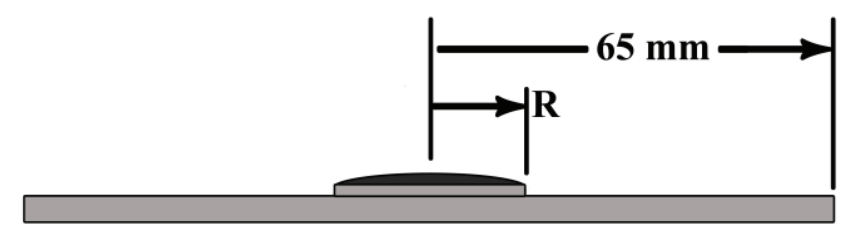

Figure 1: Section view of the substrate and drop, shown in profile. The drop, shown in black, is pinned to the edge of the circular platform that is raised slightly above the surface of the circular base.

The drops were created in a manner similar to what is reported in reference [16]. To create drops of a specified size, a pipettor or syringe was used to deposit a controlled amount of liquid on a flat circular platform that is raised slightly above the base horizontal surface, as shown in profile in Fig. 1. In this figure, the black area represents the drop. The platform is raised above the base surface in order to create a sharp circular edge to which the liquid attaches and thereby determines the size of the drop. As the drop evaporates, the contact area remains constant until eventually the drop volume is insufficient to cover the surface of the platform and the drop pulls free from the edge. All of the measurements were conducted prior to the time at which the drop pulls from the edge. In this manner, the drop radius, $\mathrm{R}$, was varied from 1 to $22 \mathrm{~mm}$ while the radius of the base horizontal surface remained constant at $65 \mathrm{~mm}$. (Note that for large radii, the term 'film' may be more appropriate than 'drop' but for consistency we use the term drop regardless of the radius.)

Initially, all of the platforms were $1 \mathrm{~mm}$ above the base surface. The platforms with a radius of $4 \mathrm{~mm}$ and smaller were lowered to $\frac{1}{2} \mathrm{~mm}$ above the base due to a concern that a geometry with a small aspect ratio of radius to elevation would influence the evaporation rate. However, the measured evaporation rates were equal for the two elevations. The platform and base are composed of a single piece of aluminum to avoid problems of poor thermal contact between the platform and base.

To prevent ambient drafts from influencing the measurements and to ensure an initially quiescent atmosphere, all experiments were contained in an enclosed volume (ca. $\left.6200 \mathrm{~cm}^{3}\right)$. Small vents were located at the base of the enclosure to allow vapor, which is heavier than air, to escape and thereby prevent it from accumulating. A comparison of measurements conducted with and without the vents indicated that the evaporation rates of large 
drops were moderately lower without the vents in the enclosure. Further investigation indicated that the reduction in the evaporation rate was due to the vapor collecting and partially filling the enclosure. For drops having a radius of $8 \mathrm{~mm}$ and smaller, no difference in the measured evaporation rates was measured with and without the vents.

Evaporation rates were measured by a simple gravimetric technique, using an analytical balance having a resolution of $0.1 \mathrm{mg}$. The balance was connected to a computer using the RS232 interface and mass data were collected at a rate of $10 \mathrm{~Hz}$. As shown in Fig. 2, the mass reduces at a constant rate, which is typical of all of the measurements except for the drops of radius equal to 1 or $2 \mathrm{~mm}$, for which the evaporation rate reduced very gradually with time, as discussed below. The evaporation rate is determined by fitting a line to the data, with the negative slope of the line equal to the evaporation rate. At the beginning of a test, some of the mass data may be unstable due to the impulse generated by the injected liquid and so the fit to the data was applied to mass values less than $90 \%$ of the maximum mass. Eventually the drop pulls away from the edge of the platform and becomes smaller, resulting in a reduction in the evaporation rate. This effect may be seen in Fig. 2 for times greater than $75 \mathrm{~s}$. To ensure that the measured evaporation rate is not influenced by data taken after the drop has pulled from the edge, the linear fit was applied to mass data greater than $50 \%$ of the maximum mass. The fact that the mass reduces at a constant rate indicates that evaporative cooling has a negligible influence.

For drops having a radius of $2 \mathrm{~mm}$ or smaller, the evaporation was slow enough that the transient gravimetric data was affected by the resolution of the analytical balance. To avoid that problem, the evaporation rates of these drops were measured using an imaging technique in which the profile of the drop was recorded at a rate of 60 frames per second. Figure 3 is an image of a $1 \mathrm{~mm} 3 \mathrm{MP}$ drop, which was acquired at the beginning of the experiment. The drop volume was calculated by integration assuming the drop is axisymmetric, and the volume was multiplied by the liquid density to obtain the drop mass. As for the gravimetric data, the evaporation rate was determined by the slope of the transient mass data. The agreement of the two measurement methods is good, as demonstrated by the data presented in Fig. 2, for which the measured evaporation rates are $0.219 \mathrm{mg} / \mathrm{s}$ for the gravimetric experiment and $0.205 \mathrm{mg} / \mathrm{s}$ for the imaging experiment. These rates differ by less than $7 \%$.

As mentioned above, for drops of radius 1 or $2 \mathrm{~mm}$, the evaporation 


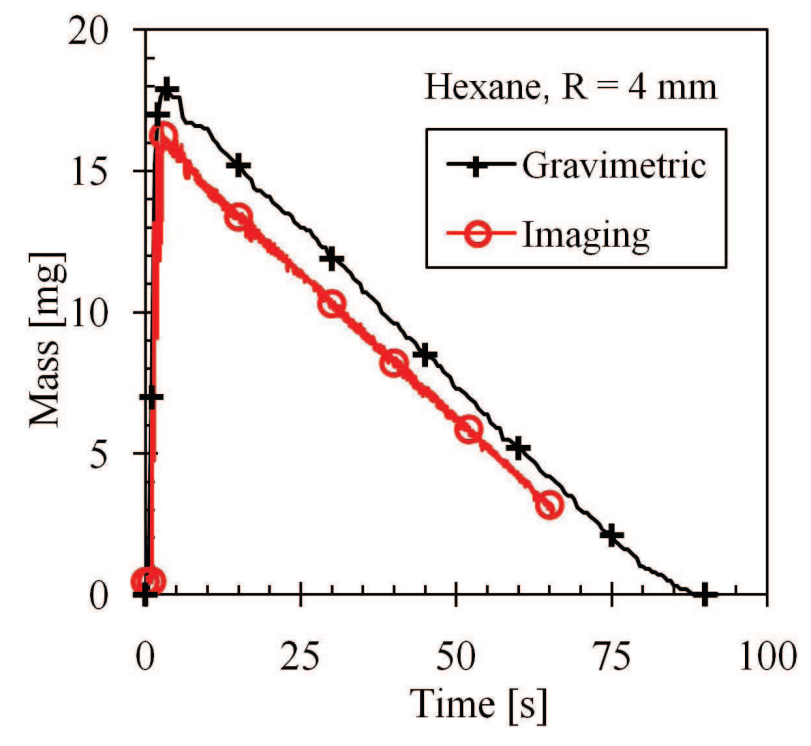

Figure 2: Plots of gravimetric and imaging data for the case of a hexane drop of radius $4 \mathrm{~mm}$. For clarity, the number of datum symbols has been greatly reduced and lines connecting the datum points are presented.

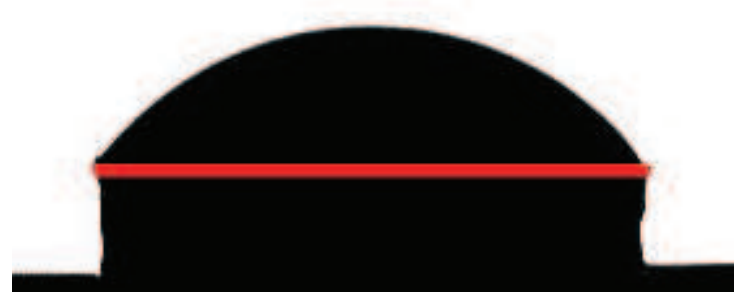

Figure 3: Image of a 3MP drop of radius $1 \mathrm{~mm}$ acquired at the beginning of the experiment. The red horizontal line indicates the surface of the platform. The contact angle is approximately $50^{\circ}$. 


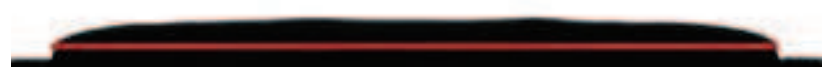

Figure 4: Image of a 3MP drop of radius $16 \mathrm{~mm}$ acquired at the beginning of the experiment. The red horizontal line indicates the surface of the platform. The contact angle is approximately $27^{\circ}$.

rate gradually decreased during the experiment. For the worst case (3MP, $\mathrm{R}=1 \mathrm{~mm}$ ) the evaporation rate varied by $20 \%$ of the median value during the experiment. Unlike for the larger drop sizes, for these small drops the contact angle varied substantially. Using Eqn. 1, which was reported by $\mathrm{Hu}$ and Larson [14], as a guide for the dependence of the evaporation rate, $E$, on the contact angle, $\theta$, a $20 \%$ variation in the evaporation rate may be due to a variation in the contact angle from $56^{\circ}$ to $10^{\circ}$, for example. The contact angles of the $1 \mathrm{~mm}$ drops generally varied from $50^{\circ}$ to $10^{\circ}$ during a measurement and so attributing the variation in the evaporation rate to the dependence on the contact angle is reasonable. In the equation, $H$ is the background vapor concentration, which is zero for our experiments, $D$ is the diffusion coefficient, and $\rho_{v}$ is the mass density of the vapor at the drop surface. Although we believe that the variation in the contact angle of the small drops may affect their evaporation rates by up to $20 \%$, the contact angle variation is much smaller in the large drops and, in fact, the influence of contact angle is not observed in the data. For example, the data presented in Fig. 2 for a hexane drop of radius $4 \mathrm{~mm}$ shows a constant evaporation rate. Figure 4 is an image of a $16 \mathrm{~mm} 3 \mathrm{MP}$ drop, acquired at the beginning of the experiment. During the experiment, the contact angle for this drop varied from $27^{\circ}$ to $21^{\circ}$. Using Eqn. 1 as a guide, this variation in the contact angle would result in less than a $2 \%$ variation in the evaporation rate.

$$
E=\pi R D(1-H) \rho_{v}\left(0.27 \theta^{2}+1.30\right)
$$

The contact angle was determined from an image by computing the slope of a line passing through the pixel at the contact point and another pixel on the surface of the drop approximately 10 pixels away. Accounting for the image resolutions, the measured contact angles were determined within 0.1 $\mathrm{mm}$ of the contact line for the $1 \mathrm{~mm}$ drop and within $0.2 \mathrm{~mm}$ for the $16 \mathrm{~mm}$ 
drop.

In comparing our measured evaporation rates to those predicted by a diffusion-limited evaporation model, we chose to use Stefan's result for evaporation from a circular plane, as reported by Gauss [17]. This form, given in Eqn. 2, accounts for the diffusion of air toward the drop surface, counter to the direction of the vapor diffusion. This counter-diffusion is accounted for by $z$, which is defined by Eqn. 3. $R_{u}$ is the universal gas constant, $T$ is the temperature, $M$ is the molar mass of the drop component and $P_{v}$ is its equilibrium vapor pressure, whereas $P_{A}$ is the atmospheric pressure. Due to the relatively high equilibrium vapor pressures of our more volatile components, especially compared with water which has been commonly studied, the inclusion of the counter diffusion term improves the solution's agreement with our measurements. $z$ varies from 1.03 for heptane to 1.13 for $3 \mathrm{MP}$ at the conditions of our experiments. By way of comparison, for a hexane drop with a contact angle of $25^{\circ}$, Eqn. 2 provides a result that is $4.3 \%$ higher than that of Eqn. 1.

$$
\begin{gathered}
E=\frac{4 R D M P_{v}}{R_{u} T} z \\
z=\frac{P_{A}}{P_{v}} \ln \frac{1}{1-\frac{P_{v}}{P_{A}}}
\end{gathered}
$$

Although the temperature variation for the experiments was small, values for the equilibrium vapor pressure and the diffusion coefficient were adjusted for temperature. Equilibrium vapor pressure was computed according to the relationships provided by Yaws [18] whereas the temperature dependence of the diffusion coefficient was computed according to Eqn. 4 [19]. $D_{o}$ is the nominal value for the diffusion coefficient, for which there is variation amongst published values, at the temperature $T_{o}$. The nominal values used in this study are listed in Table 1. Because of a relatively large variation in published values for 3MP, the value used in this study was determined by interpolation of values given in the two sources cited in Table 1.

$$
D=D_{o}\left(\frac{T}{T_{o}}\right)^{2}
$$


Table 1: Vapor pressures at $25^{\circ} \mathrm{C}$ and nominal diffusion coefficient values at temperature $T_{o}$. The vapor pressures are from [18] whereas the sources of the diffusion coefficients are given in the table.

\begin{tabular}{ccccc}
\hline \hline Component & $P_{v}[\mathrm{kPa}]$ & $D_{o}\left[\mathrm{~mm}^{2} / \mathrm{s}\right]$ & $T_{o}[\mathrm{~K}]$ & Source \\
\hline 3MP & 23.6 & 8.14 & 298 & {$[20],[21]$} \\
Hexane & 20.2 & 8.20 & 298 & {$[20]$} \\
Cyclohexane & 13.0 & 7.60 & 288 & {$[21]$} \\
Heptane & 6.11 & 7.43 & 303 & {$[22]$} \\
\hline
\end{tabular}

\section{Results and Discussion}

\subsection{Experimental results and the diffusion-controlled model}

Figure 5 presents the measured evaporation rate data for 3MP (Fig. 5a.) which is the most volatile component, and heptane (Fig. 5b) which is the least volatile component. Fits to the measured data are indicated by the solid lines and indicate the nonlinear relationship between the evaporation rate and drop radius. The data for hexane and cyclohexane follow the same trend as shown in Fig. 5. Diffusion-controlled evaporation values, computed from Eqn. 2, are also presented in the figures. For all conditions, the measured evaporation rate is greater than the diffusion-controlled rate, and the difference between the two values increases with drop radius.

To better observe how the measured evaporation rate diverges from the model for diffusion-controlled evaporation, the measured value was divided by the computed value and the quotient is called the dimensionless evaporation rate and is given the symbol $E^{*}$. The variation of $E^{*}$ with drop radius is presented in Fig. 6. If the measured values were in agreement with the model, then $E^{*}$ would be equal to 1 for all radii. As may be observed in Fig. 6, $E^{*}$ is greater than 1 and increases with drop radius for all of the components. The measured rates are between $50 \%$ and $475 \%$ greater than the values for the diffusion-controlled model. Given that Eqn. 2 gives results very close to those of Eqn. 1 and that both equations have been validated experimentally for diffusion-controlled evaporation of comparatively small drops, the results presented in Fig. 6 suggest that the diffusion-controlled evaporation model is not appropriate for large drops.

While the measured evaporation rates do not agree with calculations, it is important to note for drops having a radius up to $4 \mathrm{~mm}, E^{*}$ is approximately equal, within the bounds of uncertainty, for the four components despite their 


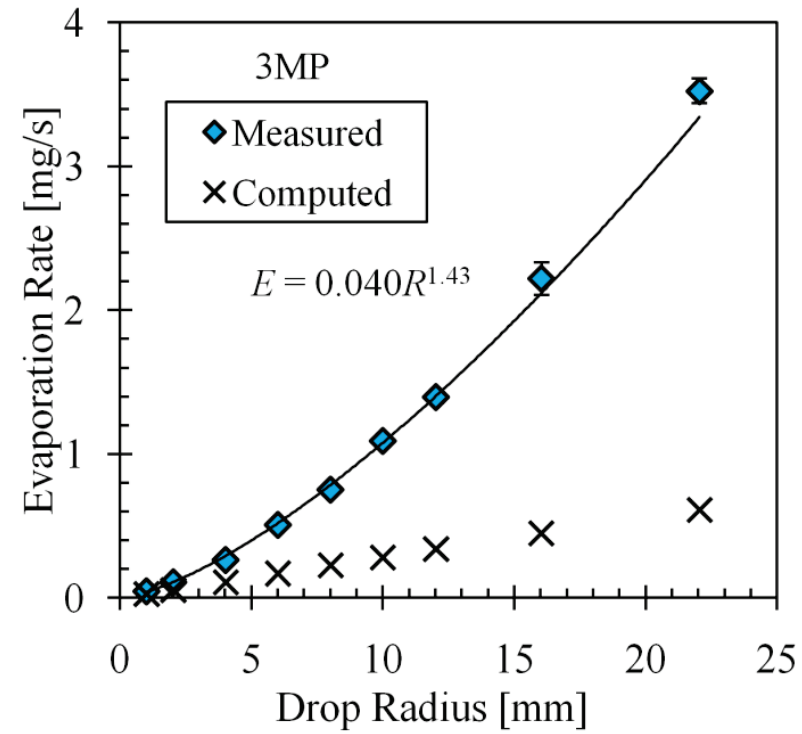

(5a)

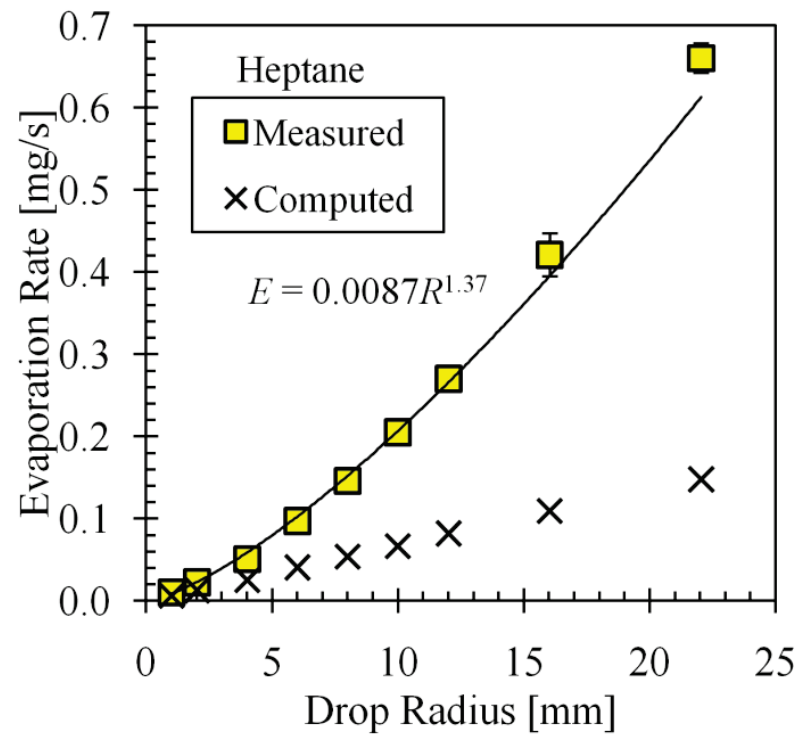

$(5 b)$

Figure 5: Plots of the measured evaporation rate as a function of drop radius. Data for 3MP, the most volatile component, is presented in Fig. 5a and data for heptane, the least volatile component, is presented in Fig. 5b. The solid line in each of the figures represents a fit to the measured data. Error bars are smaller than the data symbols except as shown. 


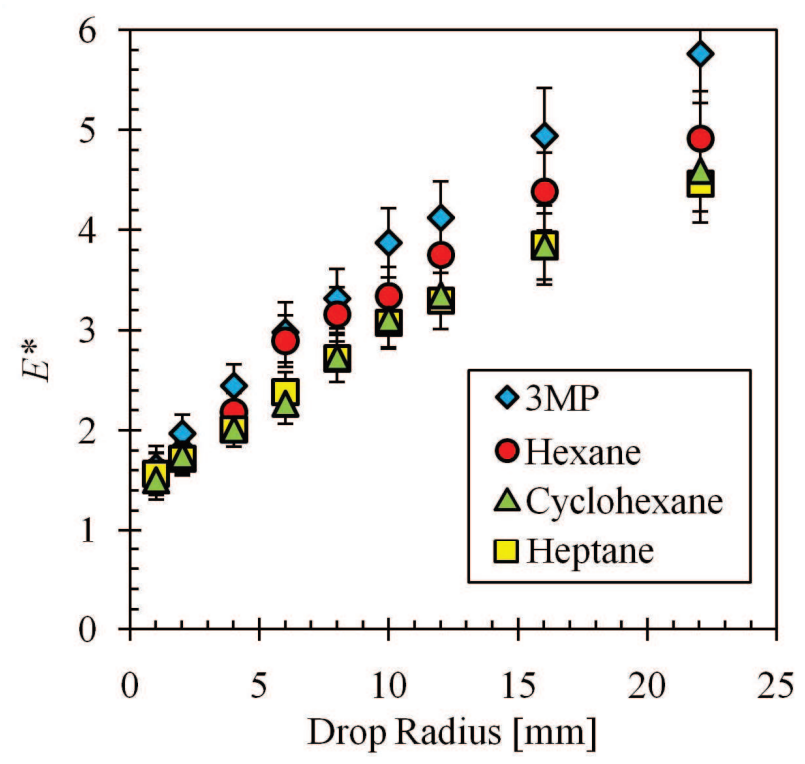

Figure 6: Plot of the dimensionless evaporation rate as a function of drop radius. $E^{*}$ equal to 1 would indicate agreement between the measured evaporation rate and that predicted by a diffusion-controlled model.

wide range of volatilities. Even at larger radii, the divergence in the trends is moderate enough that the uncertainty bounds overlap for many of the measurements. This fact suggests that whatever the phenomenon is that is causing the increased evaporation rate, it may be dependent on the same combination of physical properties that affects the rate of diffusion-controlled evaporation, $\left(M P_{v}\right) / T$.

As indicated by a previous study it is likely the vapor, which is heavier than air, flows off the surface of the drop thereby increasing the transport rate [16]. This explanation is plausible considering a comparison of representative velocity scales. The representative diffusion velocity, $v_{D}$, is equal to $D / l$, where the characteristic length, $l$, is taken to be the thickness of the vapor cloud which resides above the drop as reported in [16]. This thickness is approximately $5 \mathrm{~mm}$ for an evaporating hexane drop. The diffusion coefficient for hexane in air is approximately $8 \mathrm{~mm}^{2} / \mathrm{s}$ and so $v_{D}$ is $1.6 \mathrm{~mm} / \mathrm{s}$. To account for the largest measured discrepancy, for which $E^{*}$ is 5.8 , the characteristic convection velocity, $v_{c}$, would have to be 5.8 times greater than the diffusion velocity or $9.3 \mathrm{~mm} / \mathrm{s}$, which is a reasonable value.

The estimate of $v_{c}$ used the maximum value for the ratio of the measured- 
to-diffusive evaporation rate (5.8) and this value was obtained for a $22 \mathrm{~mm}$ radius drop of 3MP. The thickness of the vapor cloud for this drop has not been measured but is likely to be greater than the value used in the estimate for $v_{c}$. Using a larger vapor cloud thickness would result in a lower estimate of the required convection velocity and thus a less stringent test of the plausibility that natural convection is occurring during our experiments.

Natural convection is driven by a difference in densities between the vapor-air mixture and the surrounding air. A representative value for the density of the vapor-air mixture may be computed by assuming the partial pressure of the vapor is equal to the equilibrium vapor pressure, as is typically assumed at the surface of the drop. For this assumption, then, the density difference is just equal to $P_{v}\left(M-M_{a}\right) / R_{u} T$. Thus, like diffusion, natural convection is dependent upon $M P_{v} / T$, which is a dependency suggested by the trend shown in Fig. 6.

\subsection{Development of a model for combined transport}

Considering that both diffusion and convection are significant for the conditions of this study, the evaporation rate can be computed as a sum of two terms according to Eqn. 5, where $E_{d}$ represents the contribution due to diffusion and $E_{c}$ is the contribution due to convection.

$$
E=E_{d}+E_{c}
$$

Dividing Eqn. 5 through by $E_{d}$ and defining the dimensionless convection term, $E_{c}{ }^{*}$, as $E_{c} / E_{d}$ yields Eqn. 6 .

$$
E^{*}=1+E_{c}^{*}
$$

It is recognized that the vapor concentration distribution above an evaporating drop is affected by the occurrence of convection and through this effect the diffusion and convection terms are coupled. Therefore, the rate of vapor transport by diffusion from the surface of the drop would be different from the value given by Eqn. 2, and would require a computational solution. In the absence of an expression for the rate of vapor diffusion in the case of multi-mode transport, Eqn. 2 is used to estimate diffusion's contribution to the evaporation rate. For the cases in which convection is weak, for example for a component with a low vapor-air mixture density and a drop having a small radius, Eqn. 2 likely provides a good estimate of diffusion's contribution to the evaporation rate. As convection becomes stronger, the role of 


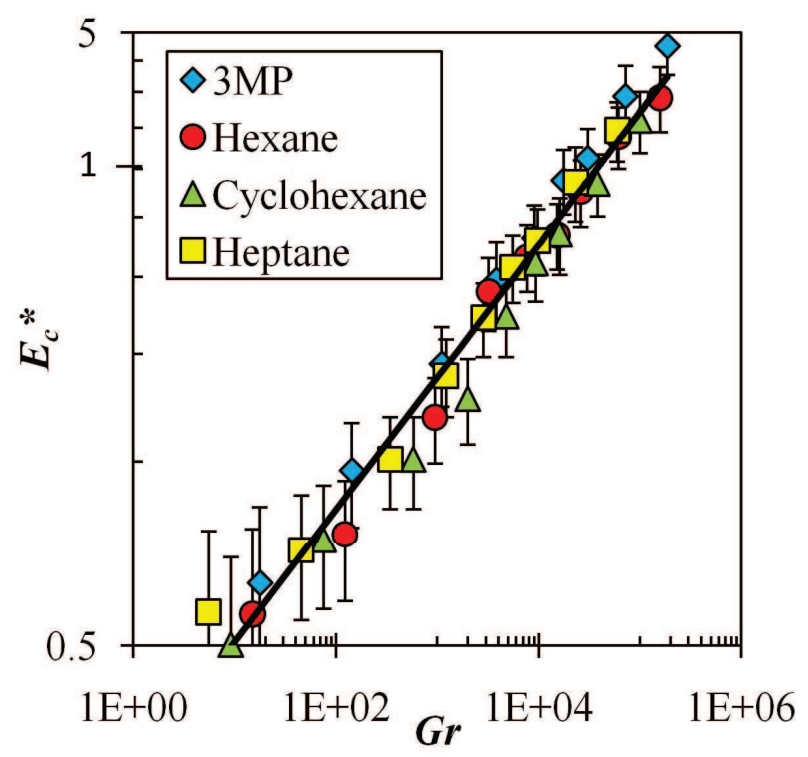

Figure 7: Plot of the dimensionless convective evaporation term as a function of the Grashof number.

diffusion becomes less significant and so inaccuracies in its estimate become less important.

To complete the model expressed by Eqn. 6, an expression is needed for $E_{c}{ }^{*}$. Since the convection is induced by buoyancy, $E_{c}{ }^{*}$ was plotted as a function of the Grashof number, $G r$, which is a dimensionless number computed according to Eqn. 7, where $\rho_{m}$ and $\rho_{a}$ are the densities of the vapor-air mixture and of air, $g$ is the gravitational constant, and $\nu_{a}$ is the kinematic viscosity of air.

$$
G r=\left(\frac{\rho_{m}-\rho_{a}}{\rho_{a}}\right)\left(\frac{g R^{3}}{\nu_{a}^{2}}\right)
$$

$E_{c}{ }^{*}$ as a function of $\mathrm{Gr}$ is presented in Fig. 7. This plot contains the data from all four components and for the complete range of drop diameters, leading to a variation in $G r$ from 5 to 186,000. The line represents a fit to all of the data, and is defined by Eqn. 8. Since $G r$ is proportional to the cube of the drop radius, Eqn. 8 indicates that the effect of convection on the evaporation rate is proportional to the drop radius raised to the power of 0.65 . 


$$
E_{c}^{*}=0.310 G r^{0.216}
$$

While the mathematical expression given by Eqn. 8 appears to fit the calculated values well, the more important question is how well does the proposed model agree with the measured evaporation rates? By combining Eqns. 2 and 5-8 and employing the ideal gas law, an equation for the evaporation rate under the conditions of combined diffusive and naturally convective vapor transport, $E_{d c}$, may be derived, which is presented as Eqn. 9. The second term in the curly brackets is the convective contribution. This term is dependent upon the product of the equilibrium vapor pressure and the molar mass, which is an expected dependency since the vapor density is directly proportional to this product.

$$
E_{d c}=\frac{4 D M P_{v} z R}{R_{u} T}\left\{1+0.310\left[\frac{P_{v} M g}{\left(P_{A}-P_{v}\right) M_{a} \nu_{a}^{2}}\right]^{0.216} R^{0.648}\right\}
$$

Figure 8 presents the difference between $E_{d c}$ and the measured evaporation rates as a function of drop size. With a few exceptions, the percentage error is within $\pm 8 \%$, and overall the root mean square error is less than $6 \%$. The model generally overpredicts the evaporation rates of cyclohexane and underpredicts the rates for 3MP. These systematic errors may be due to the values used for the diffusion coefficient, for which there is variation amongst published values.

There may be differences in the behavior of large drops compared to small ones that are not accounted for by the empirical model. For example, Marangoni-induced convection within the drop may become more significant for large drops, and this effect may cause relatively warm liquid from within the drop to flow to the surface and thereby increase the evaporation rate. However, while this possibility may contribute to an increased evaporation rate, it alone cannot account for the large difference between the measured evaporation rate and the value computed by Eqn. 2. For a $22 \mathrm{~mm} 3 \mathrm{MP}$ drop, the computed value would have to increase by a factor of 5.8 in order to equal the measured value, and this would require the temperature to increase from $23^{\circ}$ to $58^{\circ} \mathrm{C}$, an unreasonable value.

As a check of the proposed combined transport model, Eqn. 9 was used to compute the evaporation rates of methanol and acetone drops to compare with the evaporation rates of pinned drops given in references [11] and [23]. For drops ranging in size from 1 to $1.75 \mathrm{~mm}$ in radius, the results of the 


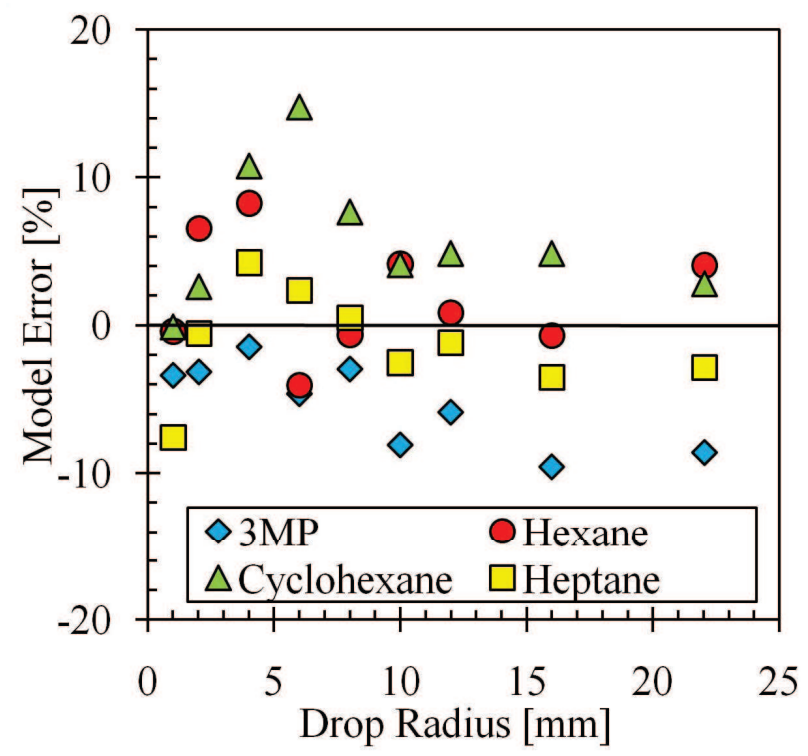

Figure 8: Plot of the percentage error in the new model as a function of drop radius. The model error is defined as $\left(E_{d c}-E_{m s d}\right) / E_{m s d}$, where $E_{m s d}$ is the measured evaporation rate. Error values for each component may be distinguished by the different data symbols.

proposed model agree fairly well with the methanol data, with the model results being about $11 \%$ higher than the measured values. For acetone, the model over predicts the measured values by about $26 \%$, which suggests a need to refine the proposed model, especially for small drops.

For the same data, it is interesting to consider how well the values computed using a diffusion-controlled evaporation equation compare with the measurements. Since these small drops have a relatively large contact angle (reportedly $43^{\circ}$ at the beginning of the experiment) the diffusion-controlled evaporation rates were computed using Eqn. 1. The computed rates are about $10 \%$ lower than the measured rates for methanol and about $21 \%$ lower than the measured rates for acetone. These results suggest, at least for acetone, that even for these small drops the evaporation rate is not being limited by diffusion for the conditions of the given experiments.

\section{Conclusions}

For the experimental conditions of this study the quasi-steady, diffusioncontrolled evaporation model was shown to be inadequate to predict the 
evaporation rates. Measured rates were from $50 \%$ to $475 \%$ greater than the values computed by the diffusion-controlled evaporation model. Based on evidence provided by [16], the higher rates are attributed to an increase in the transport of the vapor from the drop surface by natural convection.

To account for combined diffusive and convective transport, an empirical model was developed to predict the evaporation rate of a pinned, circular sessile drop having a molar mass greater than that of its atmosphere. The convective contribution to the evaporation rate was estimated by a correlation with Gr. The model assumes that the diffusive transport of the vapor is unchanged by the convective flow and that assumption implies that the distribution of the vapor concentration is unaffected by convection, which is unlikely. Despite this simplification the combined transport model agrees well with the measured evaporation rates. For components having a range of equilibrium vapor pressures that varies by a factor of four and for which the difference in densities between the vapor-air mixture and air varies by a factor of three, the model is able to predict the evaporation rate with less than $6 \%$ root mean square error.

Comparing the measured evaporation rates reported in references [11] and [23] to the values computed for diffusion-controlled evaporation supports the hypothesis that an additional transport mechanism, which we suggest is natural convection, is acting to increase the evaporation rate. Using the same data to compare with the results of the combined transport model indicates that the model over predicts the evaporation rate at the small drop sizes and suggests a need for further refinement of the model.

\section{Acknowledgement}

Acknowledgement is made to the Donors of the American Chemical Society Petroleum Research Fund for support of this research. We also gratefully acknowledge the support of the Welch Foundation.

\section{References}

[1] K. S. Lee, C. Y. Cheah, R. J. Copleston, V. M. Starov, K. Sefiane, Spreading and evaporation of sessile droplets: Universal behaviour in the case of complete wetting, Colloid Surface A 323 (2008) 63-72.

[2] C. Poulard, G. Guéna, A. M. Cazabat, Diffusion-driven evaporation of sessile drops, J Phys-Condens Mat 17 (2005) S4213-S4227. 
[3] M. Cachile, O. Bénichou, A. M. Cazabat, Evaporating droplets of completely wetting liquids, Langmuir 18 (2002) 7985-7990.

[4] M. Cachile, O. Bénichou, C. Poulard, A. M. Cazabat, Evaporating droplets, Langmuir 18 (2002) 8070-8078.

[5] C. Poulard, O. Bénichou, A. M. Cazabat, Freely receding evaporating droplets, Langmuir 19 (2003) 8828-8834.

[6] C. Poulard, G. Guéna, A. M. Cazabat, A. Boudaoud, M. B. Amar, Rescaling the dynamics of evaporating drops, Langmuir 21 (2005) 82268233.

[7] G. Guéna, C. Poulard, A. M. Cazabat, The leading edge of evaporating droplets, J Colloid Interf Sci 312 (2007) 164-171.

[8] R. D. Deegan, O. Bakajin, T. F. Dupont, G. Huber, S. R. Nagel, T. A. Witten, Contact line deposits in an evaporating drop, Phys Rev E 62 (2000) 756-765.

[9] R. D. Deegan, Pattern formation in drying drops, Phys Rev E 61 (2000) 475-485.

[10] R. D. Deegan, O. Bakajin, T. F. Dupont, G. Huber, S. R. Nagel, T. A. Witten, Capillary flow as the cause of ring stains from dried liquid drops, Nature 389 (1997) 827-829.

[11] G. J. Dunn, S. K. Wilson, B. R. Duffy, S. David, K. Sefiane, A mathematical model for the evaporation of a thin sessile liquid droplet: Comparison between experiment and theory, Colloid Surface A 323 (2008) 50-55.

[12] N. Thomas, A. Ferguson, Evaporation from a circular water surface, Philos Mag 34 (1917) 309-321.

[13] R. G. Picknett, R. Bexon, The evaporation of sessile or pendant drops in still air, J Colloid Interf Sci 61 (1977) 336-350.

[14] H. Hu, R. G. Larson, Evaporation of a sessile droplet on a substrate, J Phys Chem B 106 (2002) 1334-1344. 
[15] V. Starov, K. Sefiane, On evaporation rate and interfacial temperature of volatile sessile drops, Colloid Surface A 33 (2009) 170-174.

[16] P. L. Kelly-Zion, C. J. Pursell, R. S. Booth, A. N. VanTilburg, Evaporation rates of pure hydrocarbon liquids under the influences of natural convection and diffusion, Int J Heat Mass Tran 52 (2009) 3305-3313.

[17] A. Gauss, Fuel and Hydrocarbon Vaporization, BRL Report 1661, Aberdeen Proving Ground, Aberdeen, MD, 1973.

[18] C. L. Yaws, Thermodynamic and Physical Property Data, Gulf Publishing Company, Houston, TX, 1992.

[19] G. A. Lugg, Diffusion coefficients of some organic and other vapors in air, Anal Chem 40 (1968) 1072-1077.

[20] K. J. Beverly, J. H. Clint, P. D. I. Fletcher, Evaporation rates of pure liquids measured using a gravimetric technique, Phys Chem Chem Phys 1 (1999) 149-153.

[21] G. A. Cummings, A. R. Ubbelohde, Collision diameters of flexible hydrocarbon molecules in the vapour phase: The 'hydrogen effect', J Chem Soc (1953) 3751-3755.

[22] G. A. M. Cummings, E. McLaughlin, A. R. Ubbelohde, Collision parameters of c6-c9 hydrocarbons in the vapour phase: The hydrogen effect, J Chem Soc (1955) 1141-1144.

[23] S. David, K. Sefiane, L. Tadrist, Experimental investigation of the effect of thermal properties of the substrate in the wetting and evaporation of sessile drops, Colloid Surface A 298 (2007) 108-114. 